BY NC ND This document is the Accepted Version of Danne, N., "How to make reflectance a surface property," Studies in History and Philosophy of Modern Physics 70: 19-27. https://doi.org/10.1016/j.shpsb.2020.01.002

\title{
How to Make Reflectance a Surface Property
}

One of the commonest properties in science and everyday experience is 'reflectance', the property of car doors, mirrors, and luxury fountain pens that allows you to see your face in them (and radar systems to detect them, for that matter). Philosophers like David R. Hilbert (1987) define this property as Surface Spectral Reflectance (SSR), ${ }^{1}$ and in this essay I contend that SSR as currently defined is not a surface property, but a combination property of surface-and-medium or surface-and-light. ${ }^{2}$ What I mean by calling Hilbertian SSR a surface-and-medium property is that Hilbertian SSR is an extrinsic disposition (McKitrick 2003) of surfaces, contrary to Hilbert's (1987) claim that SSR “is an intrinsic, illumination-independent” (1177) disposition. ${ }^{3}$ To deny that Hilbertian reflectance is intrinsic will strike some as unintuitive (e.g. Isacc 2018: 521, 524; Byrne and Hilbert 2003): mirrors are supposed to remain 'reflective' objects even in the dark, just as a vase remains 'fragile' even if never dropped. By calling Hilbertian SSR an extrinsic disposition, however, I mean that light partially bestows upon mirrors their (Hilbertian) reflective capacity or reflectance profile. To the degree that this metaphysic of reflectance seems wrong,

\footnotetext{
${ }^{1}$ Hilbert continues to defend the concept of SSR as a surface property with Alex Byrne (Byrne and Hilbert 2003, 2004, 2007). Recent philosophers writing on SSR include Jackson (1998), Chirimuuta (2015), Gert (2017), and Isaac (2018).

${ }^{2}$ Any mention of "light" in this essay could be replaced with terms referring to alternative portions of the electromagnetic spectrum, such as radar or commercial radio. I argue in terms of light because Hilbert does, and because of an important problem for lased light that I explain in later sections.

${ }^{3}$ Hilbert (1987) calls SSR a 'disposition' at 386, 499, 1036, 1176, 1749, 2086 (Kindle Locations), and at Byrne and Hilbert 2003, p. 20, endnote 13. Jackson (1998) also understands SSR to be a disposition, but as supervening on a to-be-determined categorical base property (e.g. surface microstructure). I return to this categorical/dispositional distinction in the next section. Otherwise, Hilbert generally declines to defend a specific metaphysics of dispositions (tropes, universals), or to formalize the stimulus and manifestation of SSR, and so I follow suit.
} 
then Hilbert's definition requires what I show in this paper to be a somewhat mathematized reworking.

Understanding my argument requires first understanding what Hilbert means by intrinsic.

He does not precisely define the term, but his account accommodates the intuitions outlined by Jennifer McKitrick (2003):

Intuitively, a property is intrinsic if anything that has it has it regardless of what is going on outside of itself. . . Extrinsic properties, by contrast, are simply those that are not intrinsic. If a property is extrinsic, it is possible that a thing's having that property depends on what is going on outside of the thing. (McKitrick 2003: 158)

The surface of a large airplane, for example, may be considered to be its paint coat, and that paint coat can possess a weight of hundreds of pounds. But weight is an extrinsic disposition of the paint, because weight obtains and takes its definition indispensably from the terrestrial mass on which the airplane rests (e.g. Earth, Mars, the moon) (McKitrick 2003: 160). The mass of the airplane paint, on the other hand, is intrinsic to that paint (McKitrick 2003: 160), and so I would call mass but not weight a surface property obtaining at the airplane's paint coat. ${ }^{4}$ Paint weight is not a surface property, but a surface-and-planet property.

By a similar but more complex argument, I claim that Hilbertian SSR cannot be an intrinsic disposition, because Hilbert defines SSR as the disposition to reflect pulses of light. Pulses are finite-duration propagations of electromagnetic radiation, and I object that Hilbert's definition fails to account for a well-documented, empirical phenomenon of pulses that I call 'harmonic dispersion'. Harmonic dispersion is the inverse relationship of a pulse's duration to its bandwidth, and from this premise I argue that at short pulse durations, pulse-SSR (Hilbertian

\footnotetext{
${ }^{4}$ I ignore the complication that a paint coat may or may not itself have a 'surface'. Hilbert gives no metaphysic of surfaces, and so neither shall I.
} 
SSR) becomes undefined. More specifically, pulse-SSR is undefined metaphysically ${ }^{5}$ at all pulse durations, because pulse-SSR suffers a vicious infinite regress of conceptual incoherence (section 3); but because any number of operational definitions may fall short of 'welldefinedness' variously construed (e.g. the circular definition of temperature as what the thermometer says), I additionally argue the second and distinct thesis that Hilbertian SSR is not even operationally intrinsic, or intrinsic qua useable by practitioners who profitably ignore its conceptual incoherence. Granted, I do not deny that pulse-reflectance theorists succeed at their discipline and technological development; I only deny that pulse-reflectance is intrinsic to surfaces.

More positively, I argue that the disposition to reflect the harmonic components of a given pulse, components that by definition do not exhibit harmonic dispersion (see section 2), can be an intrinsic disposition in both the metaphysical and operational senses. Because a number of philosophers and scientists would reject an ontology of (Fourier) harmonics as entities literally reflecting from surfaces (Wilson 2017, Thalos 2013, McGivern 2008, Liston 1994, Redhead 1988, cf. Sheldon 1985), however, my redefinition of SSR proves non-trivial. ${ }^{6}$ I remain neutral on the question of mathematical realism (i.e. harmonic realism) in this paper, but I implicitly show that the intrinsicality requirement for reflectance brings the question to the fore. My argument proceeds in five steps. Section 1 reviews Hilbert's employment of SSR and the tacit but consequential metaphysical assumptions that he adopts in defining it. Section 2 illustrates the concepts examined in section 1, providing a short tutorial on Fourier analysis.

\footnotetext{
${ }^{5}$ By the "metaphysical" definition of a property, I mean in the very weak sense a definition free of vicious conceptual regress. I present an example of such a regress in section 3.

${ }^{6}$ As for why I decompose the pulse according to a Fourier basis instead of by some other basis such as wavelets, see section 4 , footnote 35 .
} 
Section 3 models the harmonic dispersion of a reflecting pulse to reveal why Hilbertian SSR is not a surface property, and the final two sections answer objections and conclude the paper.

\section{How Hilbert Defines and Employs SSR}

The first point to acknowledge is that Hilbert employs SSR in a theory of color perception. That is, he defines colors as sets of reflectances. Hence what I am arguing is that each individual SSR disposition that partially comprises one of Hilbert's reflectance sets cannot be a surface property, and so neither can Hilbertian surface color. Because I have nothing to say about perceptual theory, however, I focus my discussion on the individual SSR dispositions that Hilbert employs, since these dispositions are ostensibly the same kind of 'reflectance' that obtains in non-color sciences like laser physics (Stingl et al. 1995), geophysics (Gaffey 1976), radar (Haykin 1989), and others. If intrinsic pulse-reflectance is undefined in one branch of science, in other words, then I consider it undefined for any and all branches of science.

My evidence that Hilbert (1987) likewise thinks that SSR or 'reflectance' is of a univocal kind in the sciences is the following quote:

There is a well-known dispositional property of objects .... This is the surface spectral reflectance of an object. ... To measure the surface spectral reflectance . .. the ratio of the flux of incident light to the flux of reflected light is measured for each wavelength. Surface reflectances, thus conceived, are stable properties of objects. (Hilbert 1987: 1037-1041)

Thus SSR is the per-wavelength efficiency of a surface to reflect light "flux." By "flux," Hilbert means the power (in Watts) of incident or reflected light (Hilbert 1987: 1033-1042), and because light propagates sinusoidally (or as a modulated function of sine), flux is the average, as opposed 
to instantaneous power of propagating light. ${ }^{7}$ But here marks the first of Hilbert's very

consequential maneuvers. Equating flux with average power follows the standard terminology

of spectrophotometry (Germer et al. 2014), ${ }^{8}$ but electromagnetic signal theory interprets

"average power" differently. In the remainder of this section, I explain why this difference

matters to the philosophy of dispositional property ascription and of reflectance in particular.

According to signal theory, the only signals that possess average power are called "power signals," because power signals have infinite duration over which to integrate their "infinite energy"; signals with finite duration are called "energy signals" and have zero average power, because their average power, too, is computed by an integration over infinite time (Haykin and Van Veen 1999: 20-21). Thus 'energy signal' and 'power signal' are signal-theoretic names for finite-duration and infinite-duration signals, respectively. With respect to my thesis, why should any SSR theorist or spectrophotometrist care about infinite durations? No light shines on a surface for infinite duration, and if our universe originated as a Big Bang, then presumably no light could propagate to a surface for infinite duration. Hilbert and the spectrophotometrists have dispensed with signal theory to employ a shorthand version of 'average power' for finiteduration signals, and this shorthand suits their operational purposes (namely, measuring

\footnotetext{
${ }^{7}$ Said another way, the instantaneous power of a signal is a function of its instantaneous amplitude, but the instantaneous amplitude of a propagating wave changes with time and spatial location, so usually only the average power is a useful quantity. That some disciplines use hyperbolic secant or Gaussian pulses instead of sinusoidal pulses, to model the non-instantaneous ramp-up and decay of real pulse amplitudes, does not affect my argument, since pulses of all such shapes exhibit harmonic dispersion. Hence the power spectra of Gaussian and hyperbolic secant pulses resemble well enough the spectrum of a sinusoidal pulse that I use to generate the vicious conceptual regress of SSR (sections 2 and 3); for the power spectrum is a tool routinely utilized by the laser sciences to analyze electromagnetic signals (Stingl et al. 1995). I thank an anonymous reviewer for pressing me to justify this methodology in my paper.

${ }^{8}$ Spectrophotometry is one method of measuring SSR. A spectrophotometer measures the average power of light reflected from an object, by measuring electrical currents conducted by photoresistors sensitive to the optical bandwidths of interest.
} 
reflectance with hyper-picosecond pulses $^{9}$ ). For even the very lean metaphysics of Hilbertian SSR, however, this colloquialism proves devastating.

I claim that the SSR metaphysician needs infinite-duration power signals for the same reason that signal theorists do: because of the indispensability ${ }^{10}$ of power signals in accounting for harmonic dispersion (e.g. Haykin 1989: 36-37, on radar applications). I explain and illustrate harmonic dispersion in detail in the next section, but for now I need to outline the problem that harmonic dispersion creates for calling Hilbertian SSR a surface property.

Per the introduction in the previous section, harmonic dispersion is the tendency of an electromagnetic pulse, which propagates at a dominant carrier or center frequency, to propagate at more than one frequency when pulse durations become extremely short. ${ }^{11}$ This tendency, moreover, is not some kind of 'quantum problem' misapplied to the medium-sized dry goods of macroscopic science. The phenomenon is neither measurement noise nor an artifact of

\footnotetext{
${ }^{9}$ Light pulses disperse high-amplitude harmonics further than $1 \mathrm{~nm}$ from the pulse's fundamental wavelength (i.e. "center frequency" or "carrier frequency") when pulse duration falls below approximately 1 picosecond (ps, $10^{-12}$ seconds). Section 3 illustrates this phenomenon that I have already dubbed 'harmonic dispersion'. An anonymous reviewer asks why I make ' 1 ps' the threshold for metaphysically problematic dispersion, if harmonic dispersion ostensibly infects all finite-duration pulses. I answer that I choose the $1 \mathrm{~nm} / 1 \mathrm{ps}$ threshold because some applications of reflectance within Hilbertian color theory count a $1 \mathrm{~nm}$ discrepancy as moderate to severe error (e.g. Gert 2017: 69). Other, non-color applications tolerate more or less error than $1 \mathrm{~nm}$, and so the argument of this paper could be run for wider or narrower bandwidth dispersions, per application. But the point is not that color science proceeds in the sub-picosecond domain; on the contrary, Scase and Foster (1988: 196) report loss of hue discrimination in humans for exposure times below a few milliseconds. The point is that pulse-SSR conceived operationally fails when moving from color applications to sub-picosecond laser applications, and because this operational success or failure depends on pulse duration, pulse-SSR is operationally an extrinsic disposition (see section 3). That pulse-SSR also fails to be metaphysically intrinsic to surfaces, I argue in section 4.

${ }^{10}$ While Fourier analysis may be supplanted by e.g. wavelet theory, I second Liston's (1993) implicit denial that harmonic dispersion could be theoretically formulated without mathematics. See footnote 35 of this paper for further discussion of alternatives to Fourier analysis.

11 "Extreme" is a relative notion here. Light pulses propagate at hundreds of terahertz $\left(\mathrm{THz}, 10^{12} \mathrm{~Hz}\right)$ and disperse "radically" with respect to the goals of some scientific applications at durations below one picosecond. Radio waves with kilohertz carrier frequencies, on the other hand, radically disperse at millisecond durations. There is thus a billion-fold difference in what pulse durations count as "extremely" short across common electromagnetic applications.
} 
instrumentation. Harmonic dispersion is a principle of classical, Maxwellian electromagnetics (Hirlimann 2005: 31; Haykin 1989: 36-37). Thus because some long-duration pulses consist predominantly of one wavelength, but thanks to harmonic dispersion all short-duration pulses propagate as if composed of multiple wavelength components in superposition (section 2), the per-wavelength efficiency of a surface to reflect pulses (Hilbertian SSR) requires an applicationdependent minimum, non-dispersive pulse duration in order to be operationally defined (section 3). ${ }^{12}$ I therefore call Hilbertian SSR an extrinsic disposition, a surface-and-medium property instead of a surface property, because Hilbertian SSR requires for even its operational definition a specific minimum duration of the electromagnetic medium, something "going on outside of" the surface (McKitrick 2003). ${ }^{13}$ More strongly, I argue that pulse-SSR (Hilbertian SSR) is metaphysically undefined at all durations due to its colloquial appeal to 'average power' (section 3), and that calling a metaphysically undefined property an intrinsic property is implausible (section 4).

These conclusions problematize Hilbert's metaphysic of SSR for several reasons. Firstly, because they directly contradict his later work. Byrne and Hilbert (2003) claim, for example, that "the relevant physical property [to which they reduce color] must be a property of objects (more strictly, surfaces)" (9, emphasis mine). They also "rule[] out" for their color reduction the "properties an object has only if it is actually reflecting light of a specific character - for instance, light with a certain wavelength-energy distribution (spectral power distribution), or

\footnotetext{
12 "Operationally defined" means operationally exploitable, like temperature defined as what the thermometer says; operationally exploitable dispositions might not be metaphysically well-defined, and pulse-SSR is one such example. Note that a pulse duration of zero does not render SSR undefined, but only unstimulated (Hilbert 1987: Chapter 3).

${ }^{13}$ To reduce pulse duration to the duration of the surface, in hopes of recapturing the intrinsicality of SSR, would be both unprecedented and unmotivated in contemporary science. Scientists routinely change pulse duration without recording that the duration of a surface changed, unless they are recording e.g. a chemical reaction at an aging surface.
} 
wavelength composition" (9). My analysis in this paper reveals that the requirement of a minimum pulse duration above which Hilbertian SSR can be operationally defined just is the requirement for a certain "wavelength composition": namely a composition sufficiently narrow in bandwidth to enable the per-wavelength reflectance of pulses. (And if Byrne and Hilbert 2003 are not discussing a merely operational definition, then so much the worse for them, due to my arguments in section 4 against calling a metaphysically undefined property intrinsic.)

As quoted in the introduction, furthermore, to define SSR as anything but a surface property also contradicts Hilbert's (1987) earlier work. In his endeavor to reduce color to sets of SSR dispositions, Hilbert rejects what he calls the "wavelength conception of color" (Hilbert 1987: 187; hereafter WCC), or any theory that correlates color perceptions to properties of electromagnetic radiation instead of the properties of surfaces. Of course, as a color theorist, Hilbert rejects WCC for some reasons outside the scope of this paper. He reviews certain experiments, for example, that according to him undermine the case for correlating human color perceptions to properties of the electromagnetic medium (e.g. Hilbert 1987: Chapter 4). Nevertheless, because Hilbertian colors are sets of the SSR dispositions that allegedly count as 'reflectance' in all other branches of science (see my argument in the second paragraph of this section), then all of science needs to reject WCC when defining (or accepting from philosophers the concept of) dispositional reflectance. The anti-WCC constraint just is Hilbert's stipulation that SSR be intrinsic to surfaces.

Lastly, other SSR theorists who sympathize with Hilbert's color reductionism, but who argue that the categorical base of dispositional surface color is possibly (pending empirical evidence) surface microstructure (Jackson 1998: 94), will not be indifferent to the conclusion that Hilbertian SSR is not a surface property, but a surface-and-medium property. For how could 
reflectance-qua-microstructure be a shared or joint property of the electromagnetic medium? The medium contains no microstructure!

Taking stock, I have just qualitatively argued (or promised to show) that Hilbertian SSR is not a surface property, due to the pervasiveness of harmonic dispersion among propagating pulses in the sciences. I now detail the relationship of harmonic dispersion to power signals (infinite duration) and energy signals (finite duration) in the next section, to prepare my argument in section 3 that Hilbertian SSR (defined in terms of energy signals) becomes operationally undefined at short durations, and metaphysically undefined at all durations. Readers familiar with Fourier methods may profitably skip to section 3.

\section{Depicting Harmonic Dispersion, and Anticipating its Consequences}

Recall that harmonic dispersion is the widely-documented ${ }^{14}$ and empirically demonstrated inverse relationship of the duration of an electromagnetic pulse to its spectral bandwidth. Hence a preliminary step to understanding my challenge to Hilbert's concept of SSR as the intrinsic per-wavelength efficiency of a surface to reflect pulses, is to understand what kind of signals possess wavelengths. As already mentioned, such signals may be classified as energy signals (finite duration) and power signals (infinite duration).

\footnotetext{
${ }^{14}$ E.g. Hirlimann (2005); Stingl et al. (1995); Haykin (1989); Hardin (1988: 206, endnote 36). Hardin (1988) is the only philosopher of reflectance or color perception that I have seen acknowledge the existence of harmonic dispersion, although he does not employ the datum as I do in this paper.
} 
(a)

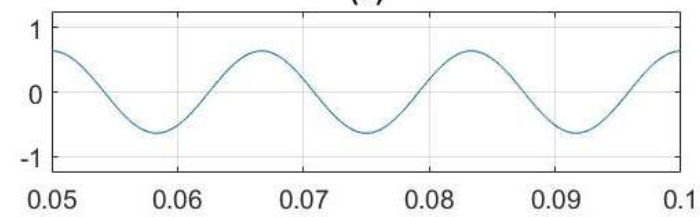

(c)

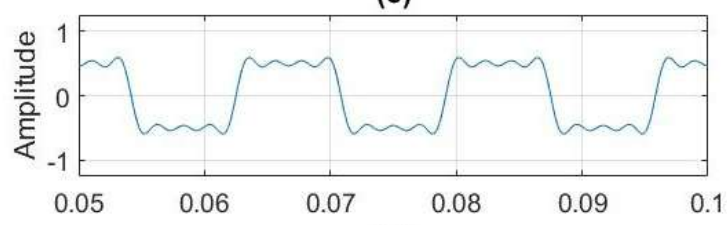

(e)

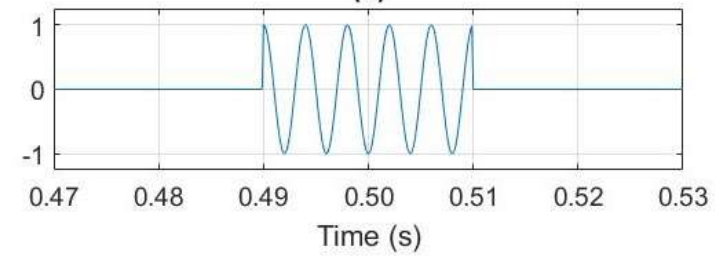

(b)

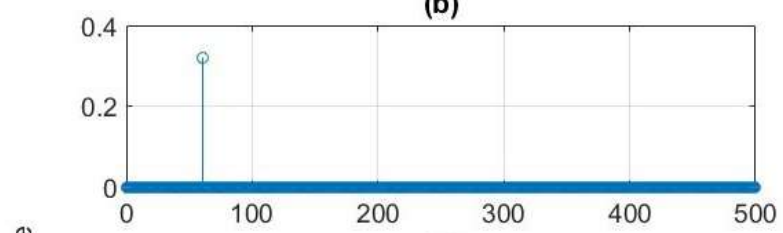

(d)
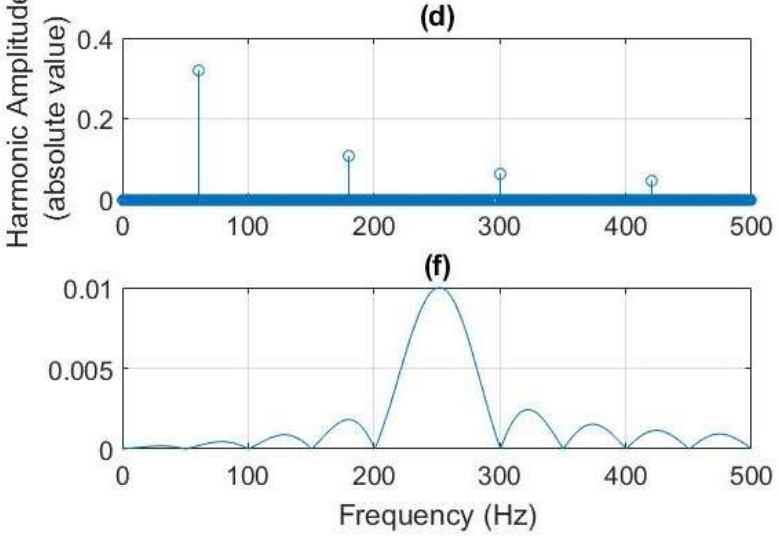

Figure 1: Superposition in the Time and Frequency Domains

Graphs (a)-(d) in Figure 1, for example, depict the power signals that superimpose to a (very rough) square wave. Graph (a) shows a $50 \mathrm{~ms}$ portion of a power signal with $60 \mathrm{~Hz}$ fundamental frequency. The units of amplitude do not matter, as they can be either electric or magnetic field strengths. Of crucial importance is the assumption that graph (a) reveals only a portion of an electromagnetic signal that possesses infinite duration. The power signal in graph (a) propagates infinitely after 0.1 seconds, and infinitely before 0.05 seconds, and so does not "start" propagating at $\mathrm{t}=0$ or $\mathrm{t}=0.05$; it has always been propagating.

The importance of the assumption that power signal (a) possesses infinite duration is that such an assumption renders power signal (a) the summation of impulses or "harmonics" in the frequency domain, as depicted in graph (b). Graph (b) reveals that power signal (a) is comprised simply of itself in the frequency domain: as a single, $60 \mathrm{~Hz}$ power signal. The $60 \mathrm{~Hz}$ signal in 
(b) superimposes with literally nothing else to become the power signal in (a). ${ }^{15}$ One advantage of such Fourier analysis (decomposition) is that it allows signal theorists to identify which frequency-domain harmonics (be they physically real or not) contribute most strongly to the time domain signal of interest. In the case of square waves, graph (d) reveals that the additional superposition of 3rd, 5th, and 7th harmonics (at $180 \mathrm{~Hz}, 300 \mathrm{~Hz}$, and $420 \mathrm{~Hz}$, respectively) change the sinusoid in (a) to a much more suggestively square signature in (c). ${ }^{16}$ Superimposing odd harmonics through the 29th or 99th index (not shown) renders the square wave very sharp indeed, with virtually no discernible ripple at the peaks (Haykin and Van Veen 1999: 178-179).

To recapitulate, graphs (a)-(d) in Figure 1 illustrate the time-domain and frequencydomain representations of power signals, which have infinite duration. Graphs (e) and (f) in Figure 1, on the other hand, depict the radically different spectral profiles possessed by energy signals, which have finite duration. The sinusoidal pulse in (e) is an energy signal, because its amplitude is zero for all times outside the window of $t=0.49$ to $t=0.51$ seconds (wherein its fundamental frequency is $250 \mathrm{~Hz}$ ). Signal (e) does not propagate for eternity, and so it is not a power signal (although (e) is the superposition of the power signals in (f)). As a consequence, the frequency-domain equivalent of (e) is not a set of discrete impulses like (b) and (d), but a continuous function of harmonic frequencies called a sinc function (graph (f)). ${ }^{17}$ This difference

\footnotetext{
${ }^{15}$ More accurately, the $60 \mathrm{~Hz}$ harmonic superimposes with a $-60 \mathrm{~Hz}$ harmonic (not shown). For clarity and transparency of applied mathematical assumptions, I omit negative-frequency harmonics from plots and break with the convention of adding their amplitudes to the plotted positive-frequency amplitudes. If someone should object that my argument lends ontological standing to negative-frequency signals, then so be it. I am not defending an ontology of mathematics in this paper, but only showing the implications of calling the reflectance disposition a surface property.

${ }^{16}$ Not every odd harmonic (3 through 7) is added to create the plot in (c). Some harmonics have negative amplitudes and therefore subtract from the superposition, but plots (b), (d), and (f) graph only the absolute values of harmonics, for aesthetic purposes.

${ }^{17}$ Haykin (1989: 18) defines $\operatorname{sinc}(x)=\sin (\pi x) / \pi x$. For the sinc function in Figure 1 (f), $x$ in the equation here given represents frequency in $\mathrm{Hz}$.
} 
between the discrete and continuous spectra of infinite and finite-duration signals proves important for my thesis. For according to graph (f), sinc function spectra exhibit peak amplitude at the fundamental frequency of the (e)-pulse $(250 \mathrm{~Hz})$, but disperse additional, non-fundamental harmonics (e.g. at $175 \mathrm{~Hz}$ and $325 \mathrm{~Hz}$ ) in proportion to the brevity or shortness of the duration of the energy signal (e). Plots (a) through (f) illustrate, in other words, the inverse relationship of harmonic dispersion: short pulses have wide spectral bandwidth, and infinitely long pulses have single-frequency or unity bandwidth.

It would be a mistake, moreover, to consider the preceding a sheerly theoretical exercise. The harmonic dispersion depicted in Figure 1 (f) finds practical application in the design of lasers (Hirlimann 2005; Stingl et al. 1995). Consider the remark by Stingl et al. (1995), for example, who identify " $[t]$ he extremely broad bandwidth necessary to generate electromagnetic energy in such short intervals" (602, emphasis mine), "short intervals" being pulse durations below 10 femtoseconds (fs; $10^{-15}$ seconds). The wide bandwidth of short pulses is a mainstay of physical optics.

Similarly important to reject is the insinuation mentioned in section 1, that harmonic dispersion (depicted in (f)) amounts to "measurement noise" or imprecision of instruments. Firstly, plots (e) and (f) are not field screen-shots, but are the input and output, respectively, of a mathematical derivation of the spectrum of a perfectly clean (noise-free, distortion-free) sinusoidal pulse. Secondly, harmonic dispersion in nature or in the laboratory is not noise, or any imperfect divergence of physical signals from theoretical signals. On the contrary, Stingl et al. (1995) find harmonic dispersion a target of fruitful "[e]xploitation" in laser science (602). That fiber-optics engineers try to compensate for, or offset various kinds of pulse dispersion in 
practice does not entail that harmonic dispersion is noise that Hilbert qua metaphysician (or alternatively, qua operationalist) can ignore in calling SSR intrinsic.

The purpose of this section has been to depict graphically what it means for a signal to reflect 'per wavelength'. While infinite-duration signals reflect exclusively at one wavelength, finite-duration signals (i.e. pulses) always reflect across an envelope of wavelengths. With this observation in hand, the metaphysics of SSR ascription can be better understood and problematized. More specifically, it can be shown that pulse-reflectance is operationally undefined for dispersive, short-durations, and metaphysically undefined at all durations. I run that argument in the next section.

\section{Why Energy-Signal Reflectance (Hilbertian SSR) Cannot be a Surface Property}

I shall now attempt to demonstrate how Hilbertian SSR (energy-signal reflectance) becomes operationally undefined at short pulse durations, and metaphysically undefined at all pulse durations. ${ }^{18}$ The sinc function that represents energy signals ${ }^{19}$ in the frequency domain, assuming time-domain amplitude $A$, duration $T$, and fundamental or carrier frequency $f_{c}$ is:

$$
G(f)=\frac{A T}{2}\left\{\operatorname{sinc}\left[T\left(f-f_{c}\right)\right]+\operatorname{sinc}\left[T\left(f+f_{c}\right)\right]\right\} \quad[1]^{20}
$$

Let $f_{c}=462 \mathrm{THz}$ represent the fundamental frequency of a pulse of light with wavelength 650 $\mathrm{nm}$. Fixing the duration of the pulse to $T=10$ femtoseconds (fs; $10^{-15}$ seconds), and the amplitude to $A=1 \mathrm{e} 12\left(1 * 10^{12}\right.$, or one trillion), permits graphic comparison with a second signal

\footnotetext{
${ }^{18}$ The results of the computer modeling that I undertake in this section closely match the physical results of laser experiments like Hirlimann (2005: 31) and Stingl et al. (1995).

${ }^{19}$ Like Figure 1 (e), in section 2.

${ }^{20}$ Equation [1] appears (with different numbering) in Haykin (1989: 37). Haykin (1989: 18) defines $\operatorname{sinc}(x)=\sin (\pi x) / \pi x$. Per footnote 15 above, I omit to plot the negative-frequency " $\operatorname{sinc}\left[T\left(f+f_{c}\right)\right]$ " component of [1] for convenience of exposition.
} 
of the same frequency, $A=1 \mathrm{e} 11$, and $T=100 \mathrm{fs}^{21}$ Figure 2 plots the positive-frequency component of [ 1 ] across a set of wavelengths utilized in laser science (Hirlimann 2005; Stingl et al. 1995), coincidentally the set of wavelengths to which human retinas are sensitive.

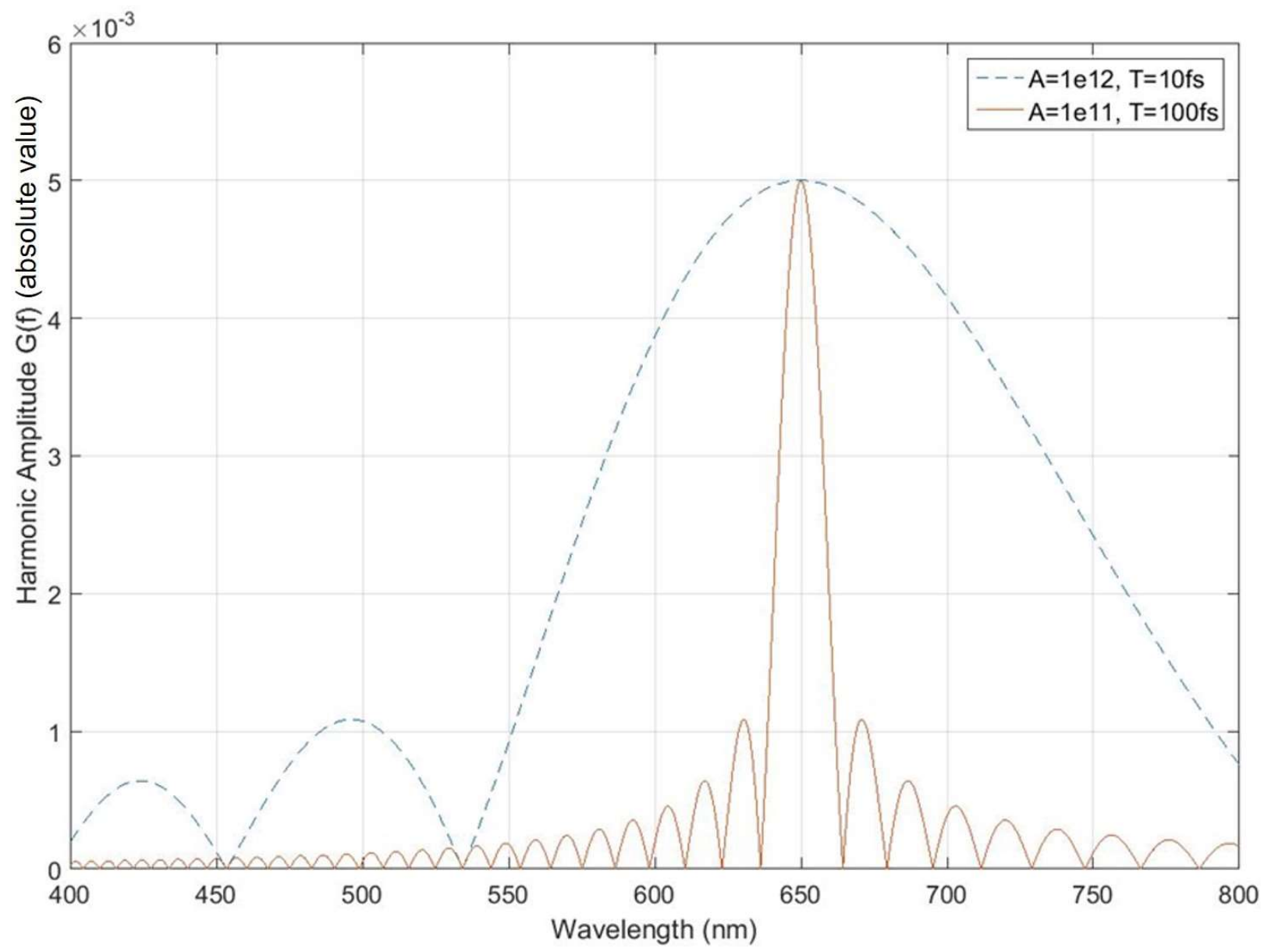

Figure 2: Fourier Decompositions of Short-Duration Pulses

The solid trace in Figure 2 is a sinc function ${ }^{22}$ representing the harmonic content of a 100 fs pulse of $650 \mathrm{~nm}$ light. Especially noteworthy are the non-zero harmonic amplitudes of what this paper calls "primary side-lobes," at 631 and $671 \mathrm{~nm}$. Hence already a problem arises for

\footnotetext{
${ }^{21}$ Note that actual trillion-volt signals are not assumed for this analysis. Amplitude numbers are chosen for aesthetic depiction in plots.

${ }^{22}$ Like Figure 1 (f), in section 2.
} 
defining SSR in terms of pulses in the highly-dispersive, sub-picosecond domain, because the pulse comprised of 631,650 , and $671 \mathrm{~nm}$ harmonics in the frequency domain enters the analysis as a $650 \mathrm{~nm}$ energy signal in the time domain.

The problem highlighted by Figure 2 materializes when philosophers ask what function an SSR plot like Figure 3 is supposed to perform. ${ }^{23}$

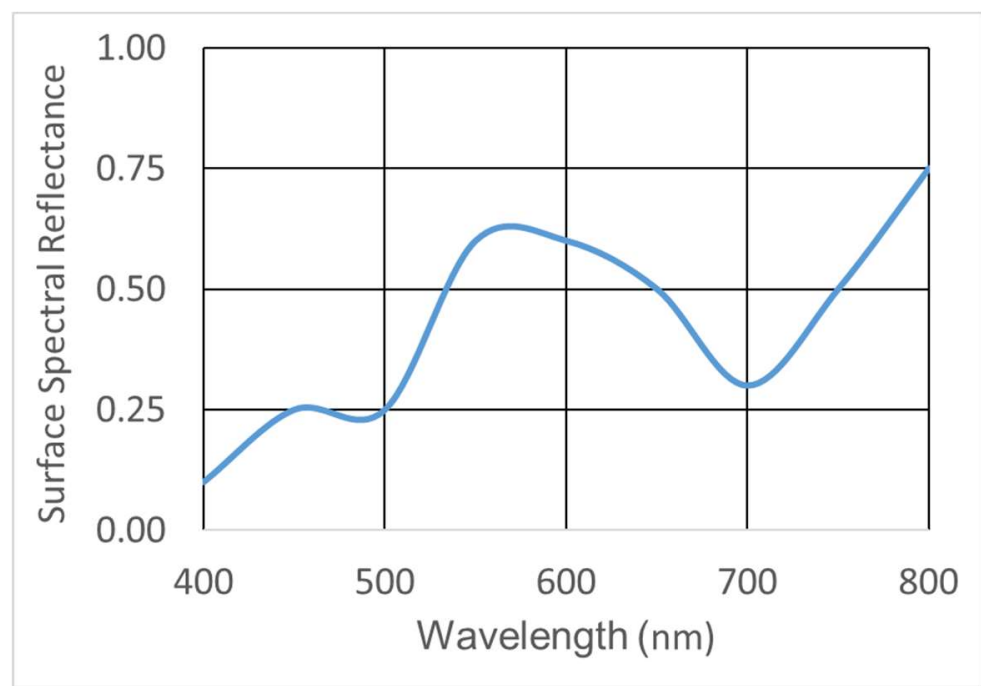

Figure 3: Hypothetical SSR Plot

Figure 3 is supposed to answer the following question: "How efficiently does this surface reflect $650 \mathrm{~nm}$ light?" The canonical response is: "Inspect the SSR plot at $650 \mathrm{~nm}$." According to Figure 3, the SSR at $650 \mathrm{~nm}$ appears to be " $50 \%$, on the nose." But Figure 2 indicates that when a surface reflects short-duration pulses of $650 \mathrm{~nm}$ light, the correct response becomes: "Inspect the SSR plot at 631,650, and $671 \mathrm{~nm}$,” which according to Figure 3 includes SSR values appreciably above and below 50\%.

\footnotetext{
${ }^{23}$ Figure 3 is contrived by me, but resembles the SSR plots found in Davies (2014), Kuehni and Hardin (2010), Churchland (2007), Pautz (2006), Byrne and Hilbert (2003), Webster (2002), Hatfield (1992), Hardin (1988), Hilbert (1987), Wandell (1985), and Gaffey (1976).
} 
The upshot is that in the sub-picosecond domain, pulse-SSR (Hilbertian SSR) values are undefined, even operationally. This undefinedness reveals itself by example. Assuming a 5 watt electromagnetic pulse generator and a perfectly reflective surface $(\mathrm{SSR}=1)$, the dotted line in Figure 2 indicates that a $10 \mathrm{fs}$ pulse of $650 \mathrm{~nm}$ light does not reflect with 5 watts at $650 \mathrm{~nm}$; a substantial portion of the reflected power instead occupies the $496 \mathrm{~nm}$ band. For simplicity, let us assume that $20 \%$ of the pulse power reflects at $496 \mathrm{~nm}$, and $80 \%$ reflects at $650 \mathrm{~nm}$. Now the question is: is the $4 \mathrm{~W}(80 \%$ of $5 \mathrm{~W}), 650 \mathrm{~nm}$ component of the original (or reflected) pulse itself a $10 \mathrm{fs}$ pulse? I answer no, lest that $10 \mathrm{fs}$ component be interpreted to disperse again, ad infinitum. There is no fact of the matter, in other words, what the SSR value of the perfect mirror is at $650 \mathrm{~nm}$, in the sub-picosecond domain; for is the power reflected at $650 \mathrm{~nm}$ equal to $4 \mathrm{~W}$, or $80 \%$ of $4 \mathrm{~W}$, or $80 \%$ of $80 \%$ of $4 \mathrm{~W}$, or $80 \%$ of $80 \%$ of $80 \%$ of $4 \mathrm{~W}$, etc.? The SSR value at 650 $\mathrm{nm}$ is undefined below durations of $1 \mathrm{ps}$, because it is totally arbitrary to determine where to stop the ' $80 \%$ regress' (see previous sentence) for the numerator and denominator of the SSR ratio. Thus there is no Hilbertian SSR value at the surface for durations below $1 \mathrm{ps},{ }^{24}$ because there is no such thing as a sub-picosecond pulse that reflects at the ostensive 'wavelength' in question $(650 \mathrm{~nm})$. To ascribe pulse-reflectance to a surface in the sub-picosecond domain is by analogy to ascribe the shape of 'circular-squareness' to that surface. The ascription is metaphysically void. Whatever reflectance disposition obtains at the mirror, it is not the disposition to reflect pulses of light. It is not Hilbertian SSR. ${ }^{25}$

Nor does it help to reply that laser scientists obviously detect a reflected, $10 \mathrm{fs}, 650 \mathrm{~nm}$ component from the perfect mirror, and that this detected 'pulse' has some measured average

\footnotetext{
${ }^{24}$ Recall that this duration threshold changes with pulse carrier frequency (see footnote 11).

${ }^{25}$ Because all finite-duration signals disperse, even long-duration signals could be said to suffer a ' $99.99 \%$ regress' akin to the ' $80 \%$ regress' above. This ' $99.99 \%$ regress' is also vicious, and so pulseSSR is metaphysically undefined for pulses of any duration. I tender this claim in section 4.
} 
power. Such a reply begs the question, and equivocates on the meaning of 'pulse'. For laser scientists know better than anyone, perhaps, that there is no such thing as a monochromatic, subpicosecond pulse in the THz range (Hirlimann 2005; Stingl et al. 1995). The option remains open, and indeed appears motivated by the preceding argument, that the disposition manifesting at the perfect mirror is the disposition to reflect the harmonics of pulses. If the laser scientist detects a $4 \mathrm{~W}$ reflection at $650 \mathrm{~nm}$, she can interpret the Fourier analysis to mean that she is measuring a $4 \mathrm{~W}, 650 \mathrm{~nm}$ harmonic. No, she cannot measure the infinitude of an infiniteduration sinusoid, but she can plausibly measure that $10 \mathrm{fs}$ portion of the harmonic that is not cancelled-out in superposition by its neighboring harmonics (see Figure $1(\mathrm{e})-(\mathrm{f})$ ).

My answer amounts to a straightforward reading of Fourier analysis and intrinsic dispositions. Unlike Hilbertian SSR, the disposition to reflect pulse harmonics per-wavelength can be intrinsic to surfaces, because that reflectance obtains despite harmonic dispersion. Thus for the non-color sciences, I recommend defining 'reflectance' (assuming it must be dispositional and intrinsic) as the disposition to reflect the harmonics of pulses. Hilbert's (1987) popular definition in terms of pulses or energy signals should be abandoned. I consider objections in the next section.

\section{Objections}

I anticipate that a number of objectors may feel the need to defend pulse-SSR (energysignal SSR or Hilbertian SSR) as a theory of color or color perception. I would find such objections misplaced. Hilbert and other color theorists already know, for example, that the loss of SSR resolution suggested by my dispersion exercise of section 3 is miniscule compared to the loss of SSR resolution to be expected from the human visual system itself (Hilbert 1987: 2281- 
2283 references the vision model of Maloney and Wandell 1986). I concede this point, and offer no claims against SSR color objectivism (Hilbert's reduction), except the intriguing insight that technically speaking, the SSR dispositions used in color objectivism are not surface properties, but surface-and-medium properties. ${ }^{26}$ For pulse-SSR is operationally defined only when impinging light is of sufficiently long duration; I illustrated this limitation with the ' $80 \%$ regress' of section 3. Yet pulse-SSR also fails to be metaphysically well-defined, for all pulse durations, because the harmonic dispersion generating the ' $80 \%$ regress' generates an equally vicious ‘99.99\% regress' for practically non-dispersive, long-duration pulses. Hence pulse-SSR does not obtain at surfaces entirely independently of impinging media, or intrinsically to a surface.

Some might nevertheless object that the disposition to reflect hyper-picosecond pulses remains intrinsic to surfaces in color theory, because in the context of color theory, subpicosecond events "going on outside of" the surface (McKitrick 2003: 158; see the introduction of this paper) do not matter to color property ascription. ${ }^{27}$ Byrne and Hilbert (2003), Hilbert (1987), and Hardin (1988: Chapter 2) all clearly explicate, for example, that correlations of human perceptual reports about color to SSR values are measured only under long-duration, stable, standard illuminants. ${ }^{28}$ So the question is how serious Hilbert (1987) is about rejecting the WCC. To the extent that the 'goings on' which differentiate intrinsicality from extrinsicality (McKitrick 2003) are vague or context-specific (e.g. time-scale dependent), then perhaps colors defined as pulse-SSR are 'intrinsic' to surfaces. My point stands, however, that pulse-reflectance

\footnotetext{
${ }^{26}$ Some color theorists, such as Pasnau (2009), seek to define color as some more-than-surfacial property. It would be premature, however, to suggest that my thesis supports or challenges any such version of color theory.

${ }^{27}$ I thank an anonymous reviewer for this objection.

${ }^{28}$ Scase and Foster (1988: 196) report a loss of hue discrimination in humans, for example, when pulses "around $500 \mathrm{~nm}$ " in wavelength fall from 1 second duration to $3 \mathrm{~ms}$ duration (pulse energy controlled).
} 
is not and cannot be the 'reflectance' (intrinsic or extrinsic) ascribed to mirrors in subpicosecond laser science, and I say more about this problem below when I answer a related objection about the optical property known as productance.

In the meantime, some may introduce trouble for my thesis by analogizing reflectance to temperature. Peter Smith (1998) argues, for example, that "there is no fact of the matter" (39) about the precise value of temperature defined as the mean kinetic energy (MKE) of substrate molecules, past a few decimal places (40-41). Why? Because that definition relies on molecular velocity, which is determined by modeling molecular motion through a hypothetical sphere, and while the sphere can shrink to a point (precisifying the meters-per-second), velocity is not "perfectly determinate" at a point (40). The Hilbertians who define SSR as pulse-reflectance could use this argument in their defense (since I claimed in section 3 that there was "no fact of the matter" about the value of pulse-SSR), except for some strong disanalogies between temperature and reflectance. Firstly, temperature is not an intrinsic property of objects or surfaces, as SSR is supposed to be. Surfaces are supposed to keep their SSR dispositions under any or no illuminant, but surfaces do not keep their temperatures under any ambient medium, except a constant one. My no-fact-of-the-matter argument about pulse-SSR (section 3) also yields more severe metaphysical implications than Smith's (1998) regarding temperature. The problem of specifying exact temperature is one of narrowing, asymptotic accuracy (many decimal places to the right), whereas the putative reflective efficiency of a perfect mirror at 650 $\mathrm{nm}$ varies widely and radically, dropping from $100 \%$ to $41 \%$ in just four iterations of the ' $80 \%$ regress' $(80 \% * 80 \% * 80 \% * 80 \% \ldots$. etc.), thus rendering the pulse-SSR value of the mirror undefined both operationally and metaphysically. ${ }^{29}$ Hence compared to the ' $80 \%$ regress' facing

\footnotetext{
${ }^{29}$ The '99.99\% regress' decreases the alleged SSR value over many iterations rather than a few iterations, but the vicious reduction of a high SSR value to a low one transpires nonetheless.
} 
pulse-SSR, Smith's (1998) metaphysical problem with temperature looks like a nice problem to have.

Perhaps a yet more cogent objection is the one suggested to me by XXXXXXX (private communication). Just as the dispositional fragility of a vase needs time to manifest—i.e. the first few nanoseconds of applied force might not stimulate the vase to break or even reveal it to be fragile - so pulse-SSR needs time to manifest its reflective efficiency. I reply that the time-tomanifest threshold between laser physics and color science proves ambiguous. The pulse duration which the color theorist needs to exceed (to avoid dispersion) is the duration that the laser scientist needs to avoid exceeding to perform useful work, and yet both parties use the same kinds of mirrors, ${ }^{30}$ and call those mirrors 'reflective' objects (Stingl et al. 1995: 603, Fig. 2). There seems to be no indication that various sciences classify different reflectances by their "attack patterns," or temporal ramp-up profiles, in the way that sounds or timbres are sometimes classified (Isaac 2018; I quote Kulvicki 2008: 7).

Hence the objection anticipated above, ${ }^{31}$ concerning Byrne and Hilbert's (2003) definition of "productance," the disposition of a surface "to produce (i.e., reflect or emit or transmit) a specific proportion of incident light" (11), such as on the surface of a translucent "stoplight..." (12). The putative challenge to my thesis is that productance is "relative to an illuminant," in Byrne and Hilbert's (2003) account, while still being an intrinsic, surface disposition. This distinction may be seen by noting that the formula for productance is " $\left(r i_{\mathrm{x}}+e\right) / i_{\mathrm{x}},{ }^{32}$ where $r$ represents the SSR value at some wavelength $\lambda, e$ is the intensity of $\lambda$ -

\footnotetext{
${ }^{30}$ In laser design, special "chirped multilayer mirrors" may be needed to condition the duration and bandwidth of a sub-picosecond pulse (Stingl et al. 1995: 602). This exception does not entail, however, that " 0.5 " reflective efficiency means something different on a chirped vs. non-chirped mirror. There is no difference in meaning.

${ }^{31}$ A version of this objection was raised to me by XXXXXX (private communication).

${ }^{32}$ Notation changed by me, namely the introduction of "x."
} 
wavelength light emitted, and $i_{\mathrm{x}}$ is the intensity of impinging radiation of wavelength $\lambda$ (from illuminant source $\left.I_{\mathrm{x}}\right)(12)$. Thus if $i_{2}$ is greater than $i_{1}$, then the productance of the surface relative to $I_{2}$ is less than the productance of the surface relative to $I_{1}$ (at the same wavelength $\lambda$ ). And yet, claim Byrne and Hilbert (2003): “Although productances are relative to illuminants, it is important to stress that the productance of a surface is illumination-independent - that is, independent of the actual illuminant" (12).

The question for my thesis, then, is: at a given wavelength $\lambda$, can a surface possess an illumination-independent disposition to reflect sub-picosecond pulses with a different (viz. nonexistent) efficiency than that at which it reflects hyper-picosecond pulses? More precisely: can one intrinsic reflectance disposition possess two illuminant-relative efficiencies, one of which is an alleged efficiency for reflecting sub-picosecond pulses? ${ }^{33}$ My answer is an emphatic no, for the simple reason that sub-picosecond pulse reflectance is undefined (section 3). I am unprepared to grant that an intrinsic property can be undefined, because I do not know what that claim would even mean. Yet further support for my conclusion hails from the discussion of laser science that transpired two paragraphs ago. Laser scientists and color scientists do not use different, time-scaled 'types' of reflective mirror (qua reflective), or give any indication that a single mirror possesses two, distinct reflective efficiencies at the same wavelength. A 0.5 reflective efficiency is 0.5 , full stop. Reflectance is not "both 0.5 and undefined" at $650 \mathrm{~nm}$. But the pulse-SSR theorist needs to claim that the pulse-SSR value at the surface of a mirror is "both 0.5 and undefined" at $650 \mathrm{~nm}$ (see section 3$)$.

Nor does my appeal to scientific practice in the preceding paragraph indicate a merely terminological dispute. ${ }^{34}$ My account remains neutral on how many intrinsic or extrinsic

\footnotetext{
${ }^{33}$ I thank an anonymous reviewer for pressing me to clarify this question.

${ }^{34}$ I thank an anonymous reviewer for this objection.
} 
dispositions can obtain at a surface, or how many of each kind of disposition the sciences should agree exist. I claim only that sub-picosecond pulse reflectance is both operationally and metaphysically undefined, and so cannot be intelligibly ascribed as intrinsic or extrinsic to any surface. I secondly claim that pulse-reflectance is metaphysically undefined for all pulse durations, and so pulse-reflectance (Hilbertian SSR) is not intrinsic to any surface. Neither of these problems is resolved by exchanging 'intrinsic' for 'extrinsic' vocabulary. I propose instead to mathematically redefine SSR from pulse-reflectance to harmonic-reflectance, since doing so renders intrinsic per-wavelength dispositional reflectance well-defined (or at least blocks the $80 \%$ and $99.99 \%$ regresses).

Especially telling in favor of my thesis is how Byrne and Hilbert (2003) construe the productance example. They define productance in terms of "monochromatic light of wavelength $\lambda \ldots$... (12). The consequences of their overlooking the energy-signal versus power-signal distinction in physical optics (section 1) could not be more salient or precipitous. Byrne and Hilbert (2003) only secure productance as an 'illumination independent' yet 'illuminant-relative' surface disposition because they are operating exclusively and by definition in the temporal domain of 'monochromatic' light: the hyper-picosecond domain. The productance example succumbs to my thesis instead of answering it, because harmonic dispersion will render the pulse-SSR component of productance (variable $r$, introduced three paragraphs ago) operationally undefined, and so productance itself operationally undefined, in the sub-picosecond domain (and metaphysically undefined for all durations). Metaphysicians of SSR in non-color sciences need to avoid this conflation of energy-signals and power-signals.

In this vein, perhaps some will think that I am abusing a mathematical idealization to infer unsupportable claims about the world, i.e., that harmonics reflect from surfaces. Pincock 
(2014) assumes without reifying an infinite depth to the oceans, for example, to simplify an equation for water wave dispersion, and Batterman (2010) claims that the taking-of-a-limit in mathematics explains certain regularities about rainbows, without reifying the limited entity (zero-wavelength rays) involved (8). Contra Pincock, I am not positing infinities to simplify a complex system or to generate a tractable equation for empirical phenomena. The world has already been described to me (by Hilbert and company) as containing a surface disposition to reflect pulses of light, and by taking that description seriously, along with empirical and mathematical insights about harmonic dispersion, I find Hilbert's metaphysic of SSR insufficient: harmonic reflectance can be a surface disposition, but pulse reflectance cannot be. Pincock (2014) needs infinitudes to describe water waves efficiently (as I might similarly predict harmonic dispersion), but he can return to the finite-ocean world because he does not obviously need to wrestle with tricky (but intuitive) dispositional constraints like the WCC. For is water wave dispersion a dispositional property? Is it 'intrinsic' to a body of water, totally independent of ambient air velocity? To impose or even consider such constraints for a descriptive project about water seems beside the point. In contrast, I posit harmonics to understand what it would take for SSR to obtain as an exclusively surfacial (intrinsic) disposition, and so I cannot as easily as Pincock relinquish my 'idealizations’.

Pace Batterman (2010: 8), moreover, I am not taking pulse duration or spectra to any absolute limit in nature, such as wavelength $\lambda=0$. I am only pointing out a pragmatic temporal lower-bound beneath which humans can no more intelligibly speak of pulse reflectance, but beneath which laser scientists nevertheless continue profitable work with what science and common sense treat as a univocal 'reflectance' property above the lower-bound. This univocity is preserved by harmonic reflectance rather than by pulse reflectance. In addition, Batterman's 
rainbow example (among others) concerns inter-theoretic reduction (Batterman 2010: 7), whereas mine does not. I argue entirely from the single science of classical, Maxwellian electromagnetics. $^{35}$

\section{Conclusion}

I have argued that dispositional reflectance, defined in terms of energy signals or 'pulses' (see sections 1 and 2) cannot be a surface property; pulse-reflectance is only definable as a surface-and-medium property or extrinsic disposition to reflect pulses whose duration exceeds a (carrier-frequency and application dependent) minimum threshold. What can be a wholly surfacial property is harmonic reflectance (section 3). The propagating harmonic (in superposition or not), due to its infinite duration, never disperses its bandwidth (see Figure 1 (a)-

${ }^{35}$ An anonymous reviewer asks why I define reflectance according to a Fourier basis of infiniteduration harmonics, when e.g. the wavelet transform (WT) correlates finite-duration pulses to finiteduration wavelet bases, and so yields fewer "non-zero" coefficients in its representation of a pulse than Fourier analysis yields. I acknowledge that Fourier analysis gives a more dramatic depiction of harmonic dispersion than the WT gives (cf. Deng et al. 2005), and that the existence of a WT option undermines the very notion that optical pulses should be physically construed as Fourier superpositions. Nevertheless, I propose moving from the Hilbertian to the harmonic definition of SSR because the latter best respects the 'per-wavelength' intuition so strongly inherent to Hilbertian SSR (see the productance argument of two paragraphs ago in the main text). Some wavelet bases, for example, are non-monochromatic on inspection, and so defining 'per wavelength' intrinsic reflectance according to them is going to be more roundabout (if feasible at all) than is simply ascribing harmonic-SSR to the surface (for a similar problem about the wavelets suitable for audio reconstruction, see Mallat 1999: 546-547).

As the same anonymous reviewer emphasizes, however, many outstanding problems remain for the intimation that pulses could be composed of real harmonics. Palmieri (2012: 533ff) objects that the human ear cannot plausibly process the high number of sinusoids in a Fourier decomposition, and Weatherall (2014) remarks that "in curved spacetime [. . . Fourier transforms are not generally welldefined" $(118$, n. 44). Referencing the same source, my anonymous reviewer mentions the existing controversy "that the 'phase velocity' of monochromatic plane-waves can exceed the speed of light in vacuum in materials with an index of refraction less than 1." I grant these points, and so declare harmonic-SSR a suitable starting-point in the metaphysical venture away from pulse-SSR, and not a unique solution for defining intrinsic reflectance, and so not an argument for (Fourier harmonic) mathematical realism, or mereological realism about superimposed harmonics. 
(b)), and so harmonic reflectance - but not pulse-reflectance — can be an intrinsic disposition to respond with a constant per-wavelength efficiency to impinging stimuli.

I interpret such harmonic reflectance to be what Byrne and Hilbert (2003) inadvertently imply to obtain at surfaces, for example when they define productance in terms of "monochromatic light..." (12). My paper reveals, however, that it is the harmonic constituents of a pulse which are monochromatic for any pulse duration, and so harmonic reflectance is (or can be) the per-wavelength disposition that SSR objectivists purport to ascribe as intrinsic to surfaces. Perhaps SSR objectivists (reflectance physicalists) prefer to contextualize the meaning of 'intrinsic' as relative to the hyper-picosecond perceptual capacities of human observers, but I have found it unclear how to square that account with Hilbert (1987) and Byrne and Hilbert (2003), both of which appear in some places to explicitly preclude such a relativization.

In the meantime, metaphysicians of the non-color sciences cannot both call SSR an intrinsic disposition, and define that disposition in terms of pulses (energy signals). Laser science, in particular, should adopt a new, harmonic definition of SSR or reflectance, to the extent that laser scientists (or philosophers) think that all mirrors possess a univocal, intrinsic reflectance property. Indeed, laser science should adopt a harmonic redefinition of reflectance even if scientists and philosophers deny that reflectance is univocal or intrinsic, because subpicosecond pulse-reflectance is not only metaphysically undefined, but even operationally so. 


\section{References:}

Batterman, Robert W. 2010. "On the Explanatory Role of Mathematics in Empirical Science." Brit. J. Phil. Sci. 61: 1-25.

Byrne, Alex and David R. Hilbert. 2003. "Color Realism and Color Science.” Behavioral and Brain Sciences 26: 3-64.

---. 2004. "Hardin, Tye, and Color Physicalism." The Journal of Philosophy 101 (no. 1): 37-43.

---. 2007. “Truest Blue.” Analysis 67 (no. 1): 87-92.

Chirimuuta, Mazviita. 2015. Outside Color: Perceptual Science and the Puzzle of Color in Philosophy. Cambridge, MA: The MIT Press.

Churchland, Paul. 2007. "On the Reality (and Diversity) of Objective Colors: How Color-Qualia Space Is a Map of Reflectance-Profile Space.” Philosophy of Science 74 (no. 2): 119-149.

Davies, Will. 2014. “The Inscrutability of Color Similarity.” Philosophical Studies 171: 289-311.

Deng, Yuqiang, Zubin Wu, Lu Chai, Ching-yue Wang, Keisaku Yamane, Ryoji Morita, Mikio Yamashita, and Zhigang Zhang. 2005. "Wavelet-transform analysis of spectral shearing interferometry for phase reconstruction of femtosecond optical pulses." Optics Express 13: $2120-2126$.

Gaffey, Michael J. 1976. "Spectral Reflectance Characteristics of the Meteorite Classes." Journal of Geophysical Research 81 (no. 5): 905-920.

Germer, Thomas A., Joanne C. Zwinkels, and Benjamin K. Tsai (Eds.). 2014. Spectrophotometry: Accurate Measurement of Optical Properties of Materials. Waltham, MA: Elsevier Inc.

Gert, Joshua. 2017. Primitive Colors: A Case Study in Neo-pragmatist Metaphysics and Philosophy of Perception. New York: Oxford University Press.

Hardin, C. L. 1988. Color for Philosophers. Indianapolis: Hackett Publishing Company.

Hatfield, Gary. 1992. "Color Perception and Neural Encoding: Does Metameric Matching Entail a Loss of Information?" PSA: Proceedings of the Biennial Meeting of the Philosophy of Science Association, Volume One: Contributed Papers, 492-504.

Haykin, Simon. 1989. An Introduction to Analog and Digital Communications. New York: John Wiley \& Sons, Inc.

Haykin, Simon and Barry Van Veen. 1999. Signals and Systems. New York: John Wiley \& Sons, Inc. 
Hilbert, David R. 1987. Color and Color Perception: A Study in Anthropocentric Realism. Center for the Study of Language and Information, Stanford University. Kindle Edition.

Hirlimann, C. 2005. "Pulsed Optics." In Femtosecond Laser Pulses: Principles and Experiments, 2nd edition, edited by Claude Rulliere. Berlin: Springer-Verlag.

Isaac, Alistair M. C. 2018. "Prospects for Timbre Physicalism.” Philosophical Studies 175: 503529.

Jackson, Frank. 1998. From Metaphysics to Ethics. Oxford: Clarendon Press.

Kuehni, Rolf G. and C. L. Hardin. 2010. "Churchland's Metamers.” British Journal for the Philosophy of Science 61, 81-92.

Kulvicki, John. 2008. “The Nature of Noise.” Philosopher's Imprint 8 (no. 11): 1-16.

Liston, Michael. 1994. "How Abstract Objects Strike Us.” Dialectica 48 (no. 1): 3-27.

---. 1993. "Taking Mathematical Fictions Seriously.” Synthese 95 (no. 3): 433-458.

Mallat, Stéphane. 1999. A Wavelet Tour of Signal Processing, 2nd Edition. San Diego: Academic press.

Maloney, Laurence T., and Wandell, Brian A. 1986. "Color constancy: a method for recovering surface spectral reflectance.” J. Opt. Soc. Am. A 3 (no. 1): 29- 33.

McGivern, Patrick. 2008. "Reductive Levels and Multi-Scale Structure.” Synthese 165: 53-75.

McKitrick, Jennifer. 2003. "A Case for Extrinsic Dispositions.” Australasian Journal of Philosophy 81 (no. 2): 155-174.

Palmieri, Paolo. 2012. "Signals, cochlear mechanics and pragmatism: a new vista on human hearing?" Journal of Experimental \& Theoretical Artificial Intelligence 24 (no. 4): 527545.

Pasnau, Robert. 2009. “The Event of Color.” Philosophical Studies 142: 353-369.

Pautz, Adam. 2006. "Can the Physicalist Explain Colour Structure in Terms of Colour Experience?” Austraulasian Journal of Philosophy 84 (no. 4): 535-564.

Pincock, Christopher. 2014. "How to Avoid Inconsistent Idealizations." Synthese 191: 29572972.

Redhead, M. 1988. "A philosopher looks at quantum field theory." In Philosophical foundations of quantum field theory, edited by H. R. Brown and R. Harré. Oxford: Clarendon Press. 
Scase, Mark O. and David H. Foster. 1988. "Anomalous loss in blue-green wavelength discrimination with very brief monochromatic stimuli presented to the normal human eye." Ophthal. Physiol. Opt., Vol. 8 (April).

Sheldon, Neil A. 1985. "One Wave or Three? A Problem for Realism." British Journal for the Philosophy of Science 36 (no. 4): 431-436.

Smith, Peter. 1998. Explaining Chaos. Cambridge: Cambridge University Press.

Stingl, A., M. Lenzner, Ch. Spielmann, F. Krausz, and R. Szipocs. 1995. "Sub-10-fs mirrordispersion-controlled Ti:sapphire laser." Optics Letters 20 (no. 6): 602-604.

Thalos, Miriam. 2013. Without Hierarchy. New York: Oxford University Press.

Wandell, Brian A. 1985. "The Synthesis and Analysis of Color Images.” NASA Technical Memorandum 86844.

Weatherall, James Owen. 2014. "Against dogma: On superluminal propagation in classical electromagnetism." Studies in History and Philosophy of Modern Physics 48: 109-123.

Webster, W. R. (2002). "Wavelength Theory of Colour Strikes Back: The Return of the Physical." Synthese 132 (no. 3): 303-334.

Wilson, Mark. 2017. Physics Avoidance: Essays in Conceptual Strategy. New York: Oxford University Press. 